\title{
Axial length, refraction, and retinal vascularization I year after ranibizumab or bevacizumab treatment for retinopathy of prematurity
}

This article was published in the following Dove Press journal:

Clinical Ophthalmology

2I July 2016

Number of times this article has been viewed

Chun-Ju Lin ${ }^{1,2}$

Yi-Yu Tsai ${ }^{1,2}$

'School of Medicine, College of Medicine, China Medical University, Taichung, Taiwan; ${ }^{2}$ Department of Ophthalmology, China Medical University Hospital, China Medical University, Taichung, Taiwan
Correspondence: Chun-Ju Lin Department of Ophthalmology, China Medical University Hospital, No 2,

Yuh-Der Road, Taichung, Taiwan 40447, Republic of China

Tel +886 $422052|2|$ ext | | 4 |

Fax +886422059265

Email doctoraga@gmail.com
Purpose: The purpose of this study was to report on the axial length, refraction, and retinal vascularization 1 year after ranibizumab or bevacizumab treatment for threshold retinopathy of prematurity.

Methods: The authors conducted a comparative, consecutive, original study.

Results: Twenty-five eyes of 13 patients with threshold retinopathy of prematurity received one intravitreal ranibizumab treatment, and 15 eyes of eight patients received one intravitreal bevacizumab treatment. In the ranibizumab group, the mean gestational age was $26.15 \pm 2.08$ weeks, with a mean birth weight of $811.15 \pm 287.3 \mathrm{~g}$. In the bevacizumab group, the mean gestational age was $26.50 \pm 2.14$ weeks, with a mean birth weight of $938.38 \pm 200.4 \mathrm{~g}$. The mean axial length was $20.34 \pm 0.97 \mathrm{~mm}$ and the mean spherical equivalent was $0.46 \pm 1.36 \mathrm{D}$ in the ranibizumab group, with complete vascularization in 15 of $25(60 \%)$ eyes. The mean axial length was $20.91 \pm 1.54 \mathrm{~mm}$ and the mean spherical equivalent was $-0.60 \pm 3.86 \mathrm{D}$ in the bevacizumab group, with complete vascularization in seven of $15(46.7 \%)$ eyes.

Conclusion: There were no significant differences in the axial length and refraction between children with threshold retinopathy of prematurity who received intravitreal bevacizumab compared to those who received ranibizumab after 1 year of follow-up. It appeared that the ranibizumab treatment could achieve more complete retinal vascularization than the bevacizumab treatment; however, there was no statistical significance and long-term follow-up is needed.

Keywords: bevacizumab, ranibizumab, refraction, retinal vascularization, retinopathy of prematurity, axial length

\section{Introduction}

Threshold retinopathy of prematurity (ROP) is defined as zone I ROP (any stage of ROP with plus signs or stage 3 ROP with no plus signs) or zone II ROP (stage 3 ROP with plus disease and neovascularization [NV] for more than 5 contiguous or 8 interrupted clock hours). ${ }^{1}$ Since anti-VEGF treatment for ROP has gained wider acceptance, ${ }^{2-7}$ ophthalmologists have become concerned about the refractive status after treatment. In this study, we reported the axial length and refraction 1 year after ranibizumab (Lucentis ${ }^{\circledR}$; Hoffman-La Roche Ltd., Basel, Switzerland) or bevacizumab (Avastin ${ }^{\circledR}$; Genentech/Roche) treatment for threshold ROP and compared the differences between the two groups.

\section{Methods}

The authors conducted a comparative, consecutive, original study. Patients with threshold ROP were identified and evaluated retrospectively from August 2011 to 
January 2012. The diagnosis was based on the guidelines of the International Committee for the Classification of ROP. ${ }^{1}$ The Institutional Review Board of China Medical University Hospital approved the study protocol. The study was performed in accordance with the World Medical Association's Declaration of Helsinki.

Informed oral and written consent was obtained from the parents of the patients. For the use of anti-VEGF agents, we offered both bevacizumab and ranibizumab as treatment options. Detailed information regarding single-dose ranibizumab and single-dose bevacizumab was provided. We specifically emphasized that both were off-label use for ROP, but ranibizumab is designed specifically for intravitreal administration with a possibly higher safety profile. The parents were required to pay for the drug. The main disadvantage of using ranibizumab is that it is much more expensive than bevacizumab.

All infants received intravitreal injections of ranibizumab ( $0.25 \mathrm{mg} / 0.025 \mathrm{~mL}$; half dose of adult based on experience) with bevacizumab $(0.625 \mathrm{mg} / 0.025 \mathrm{~mL})$ in the literature. ${ }^{2-7}$ All patients received one intravitreal ranibizumab injection or one bevacizumab injection under topical anesthesia as the only treatment. Levofloxacin (Cravit; Santen Pharmaceutical Co., Ltd., Osaka, Japan) eyedrops were given four times daily for 1 week.

In this study, patients who needed further injections or ablative therapy were excluded, because the main aim of our study was to find out the effects of the single intravitreal ranibizumab or bevacizumab treatment on axial length, refraction, and retinal vascularization. The cases that needed further ablative therapy were also excluded due to the possibly greater impact on refractive errors.

The follow-up examination at 1 year after treatment included axial length measurement and automated refractometry under cycloplegic conditions. An examination of the anterior and posterior segments of the eye was also performed by one senior retinal subspecialist (C-J Lin). No original treated patients were lost to follow-up.

A contact method A-scan biometer (Sonomed A2500; Ophthalmic Instrument Co., Inc., Avon, MA, USA) was used to determine the axial length (in $\mathrm{mm}$ ), which was defined as the distance between the anterior surface of the cornea and the fovea. In each case, the axial length was measured under topical anesthesia with $0.5 \%$ proparacaine hydrochloride (Alcaine; Alcon Laboratories, Inc., Fort Worth, TX, USA). The probe was placed against the cornea with as little pressure as possible, and an average of five measurements was used.
A Hand Held Refkeratometer Retinomax K-plus2 instrument (Nikon Instruments, Melville, NY, USA) was used to objectively determine the spherocylindrical refractive error. Tropicamide $0.5 \%$ /phenylephrine $0.5 \%$ (Mydrin-P; Santen, Osaka, Japan) was used for pupillary dilatation and cycloplegia prior to refraction. The refraction procedure was performed by the same optometrist. Objective refraction was measured using the Hand Held Refkeratometer Retinomax K-plus2 instrument under topical anesthesia and converted into a spherical equivalent (defined as spherical errors and half of the astigmatic errors) expressed in diopters.

The authors minimized study errors by only including patients who received one intravitreal injection. In addition, the averages of at least five measurements of both axial length and refraction were used in the analysis. The same instruments were used by the same optometrist in each case, and all patients received the same treatment regimen and follow-up.

The authors collected patient data and analyzed them as follows. Differences between data in two groups of treatment were compared with specific statistical methods. For patientrelated data, such as sex, gestational age, and birth weight, Fisher's exact test was used for categorical variables and Student's $t$-test was used for continuous variables. For eyerelated data, such as stage, zone, axial length, and percentage of patients with full vascularization, methods for generalized linear mixed model (GLMM) were used. This kind of model can control the random effect of clustered data (such as the two eyes from the patient in either group in our case) and avoid the bias resulting from the dependency of data. PROC GLIMMIX is a GLMM procedure in SAS statistical software (SAS Institute Inc., Cary, NC, USA), which can be applied, no matter whether for categorical or continuous dependent variable, and is thus very flexible. We applied this procedure in our study for clustered data.

\section{Results}

Twenty-five eyes of 13 patients who received intravitreal ranibizumab were included. Of these 25 eyes, eight were zone I and 17 were zone II stage 3 ROP. The mean gestational age was 26.15 \pm 2.08 weeks (range: 24-30 weeks), with a mean birth weight of $811.2 \pm 287.3 \mathrm{~g}$ (range: $507-1,480 \mathrm{~g}$ ) and a mean postmenstrual age at intravitreal injection of 35.15 \pm 1.994 weeks (range: $32-39$ weeks).

Fifteen eyes of eight patients who received intravitreal bevacizumab were included. Of these 15 eyes, two were zone I, eleven zone II, and two zone III stage 3 ROP. The mean gestational age of these patients was $26.50 \pm 2.14$ weeks (range: 24-30 weeks), with a mean birth weight of 938.4 $\pm 200.4 \mathrm{~g}$ 
(range: $724-1,247 \mathrm{~g}$ ) and a mean postmenstrual age at intravitreal injection of 38.25 \pm 4.166 weeks (range: $32-46$ weeks).

All eyes received only one intravitreal injection as the primary therapy and all showed regression of retinal NV. No significant systemic complications were noted. No cataracts, endophthalmitis, or retinal detachment occurred postoperatively. Preretinal hemorrhages were found in four eyes (16\%) of three patients in the ranibizumab group; however, these were absorbed after 11-14 weeks without sequelae.

In the ranibizumab group, the mean axial length was 20.34 $\pm 0.97 \mathrm{~mm}$ (range: 18.65-22.06 $\mathrm{mm}$ ) and the mean spherical equivalent was $0.46 \pm 1.36 \mathrm{D}$ (range: $-2.38-4.13 \mathrm{D}$ ). Complete vascularization toward the retinal periphery was noted in 15 of $25(60 \%)$ eyes. In the bevacizumab group, the mean axial length was $20.91 \pm 1.54 \mathrm{~mm}$ (range: 19.53-24.83 mm) and the mean spherical equivalent was $-0.60 \pm 3.86 \mathrm{D}$ (range: -10.63-4.50 D). Complete vascularization toward the retinal periphery was noted in seven of 15 (46.7\%) eyes.

We separated the patient-specific analysis and eyespecific analysis into two different parts in Table 1. There were no statistically significant differences in the sex, mean gestational age, mean birth body weight, and mean postmenstrual age at intravitreal injection between the two groups (Table 1). In addition, there were no statistically significant differences in the number of eyes, laterality, eyes with different ROP zones, mean axial length, mean spherical equivalent, and status of vascularization toward

Table I Comparison of the sex, mean gestational age, mean birth weight, mean postmenstrual age at intravitreal injection, and eyes with different ROP zones

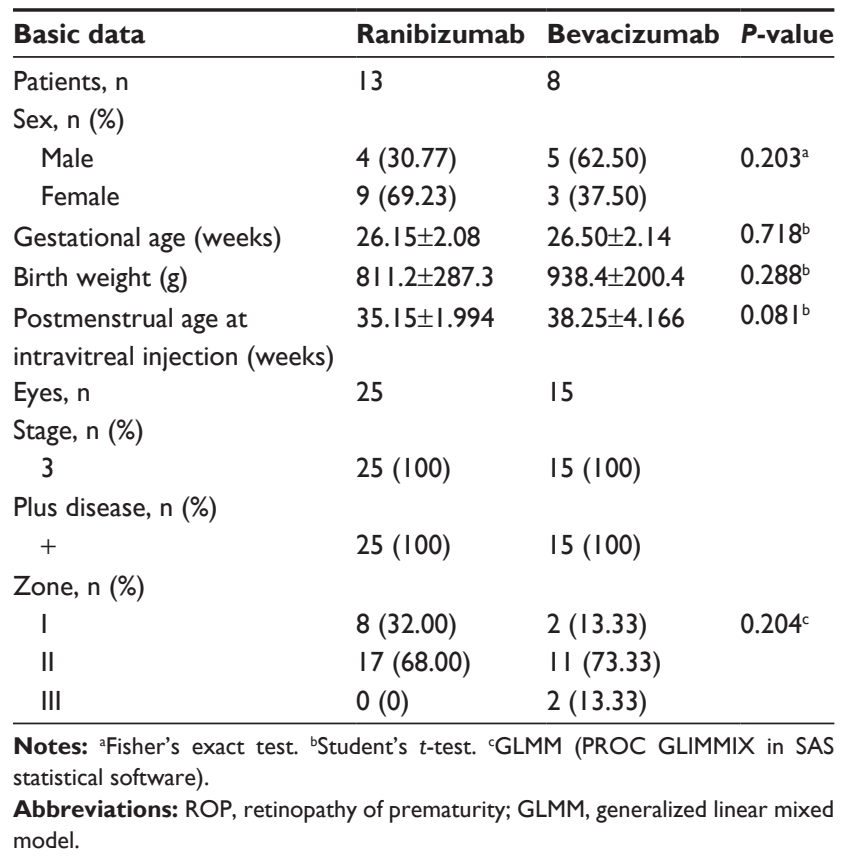

Table 2 Comparison of the number of eyes, laterality, mean axial length, mean spherical equivalent, and status of vascularization toward the retinal periphery after I year of follow-up

\begin{tabular}{llll}
\hline One-year data & Ranibizumab & Bevacizumab & P-value \\
\hline Eyes, $\mathrm{n}$ & 25 & 15 & \\
Eye, $\mathrm{n}(\%)$ & & & \\
$\quad$ OD & $13(52)$ & $7(46.67)$ & $0.748^{\mathrm{a}}$ \\
$\quad$ OS & $12(48)$ & $8(53.33)$ & \\
Axial length (mm) & $20.34 \pm 0.97$ & $20.91 \pm 1.54$ & $0.287^{\mathrm{a}}$ \\
Spherical equivalent (D) & $0.46 \pm 1.36$ & $-0.60 \pm 3.86$ & $0.357^{\mathrm{a}}$ \\
Full vascularization, $\mathrm{n}(\%)$ & & & \\
$\quad$ Yes & $15(60)$ & $7(46.7)$ & $0.602^{\mathrm{a}}$ \\
$\quad$ No & $10(40)$ & $8(53.3)$ & \\
\hline
\end{tabular}

Note: a'GLMM (PROC GLIMMIX in SAS statistical software).

Abbreviations: OD, right eye; OS, left eye; GLMM, generalized linear mixed model.

the retinal periphery at 1-year follow-up between the two groups (Table 2).

\section{Discussion}

The treatment modalities of threshold ROP include cryotherapy (published in a clinical trial in 1988), ${ }^{8}$ laser therapy (published in a clinical trial in 2003), ${ }^{9}$ and intravitreal bevacizumab (published in a clinical trial in 2011). ${ }^{2}$ However, cryotherapy involves scarring of the full ocular thickness, and laser therapy involves scarring of the retina and choroid. The main concerns following these peripheral retinal ablative procedures are inflammation of the eyes and induced high refractive errors or astigmatism. ${ }^{10-14}$

Vitreous fluid VEGF has been found to be highly elevated in patients with ROP, ${ }^{15}$ and NV is mainly attributable to the expression of VEGF. Treatment with an anti-VEGF agent such as bevacizumab and ranibizumab is effective and appears to be well tolerated in the majority of cases with ROP, especially in cases of stage 3 ROP..$^{2-7}$ Nevertheless, long-term surveillance is necessary to clarify whether ocular or systemic side effects eventually occur. In addition, there are also concerns about the refractive status after anti-VEGF treatment. We therefore conducted this study to record the axial length and refraction 1 year after ranibizumab or bevacizumab treatment for threshold ROP, and then compared the differences between the two groups.

We found that intravitreal ranibizumab or bevacizumab used as the primary treatment for ROP led to NV regression in all patients with threshold ROP. The plus signs also decreased after the injections. Preretinal hemorrhages were found in some eyes after the injection; however, they were all absorbed without sequelae. No apparent systemic adverse effects related to the injections were noted, and no eyes needed conventional laser photocoagulation or cryotherapy 
as an adjuvant therapy. Studies regarding the effects and complications associated with intravitreal ranibizumab injections for threshold ROP remain scarce. Jang et al ${ }^{16}$ reported a case of delayed-onset retinal detachment after laser photocoagulation and an intravitreal ranibizumab injection for zone 1 plus ROP. Another prospective, experimental, longitudinal, and open study of threshold-prethreshold ROP or plus disease included cases receiving combined laser-ranibizumab therapy who were followed up for 3 years. ${ }^{6}$ Regression of retinopathy was noted in all cases. However, $17.6 \%$ showed persistence of vascular tortuosity without vascular dilatation, and $11.7 \%$ showed vitreous membrane development. In such cases, laser therapy may scar the peripheral retina, cause intraocular inflammation, and induce preretinal membrane formation.

On the other hand, intravitreal ranibizumab was found to be useful as a salvage therapy in a case of rush-type ROP. ${ }^{17}$ In this case, extraretinal fibrovascular proliferation persisted despite intravitreal bevacizumab and laser photocoagulation. However, after intravitreal ranibizumab treatment, a fundus examination showed flat retinas and normal vasculature during 2 years of follow-up. Lin et $\mathrm{al}^{7}$ also reported the effects of a single intravitreal ranibizumab injection in the treatment of very low birth weight infants with ROP. In this preliminary report, all cases showed regression of ROP without vascular tortuosity or vitreous membrane formation, which may be complicated by laser therapy.

Four eyes of three patients had postoperative preretinal hemorrhages at the border of the vascular and avascular retinas in our ranibizumab group. Ranibizumab can lead to the regression of new vessels, and the involved forces exerted on the NV may lead to local bleeding. The hemorrhages did not cause significant ocular damage and were absorbed after 11-14 weeks without sequelae. The rate of preretinal hemorrhage in our series was $16 \%$, which is higher than the report of $\mathrm{Wu}$ et $\mathrm{al}^{3}(9.76 \%)$. The possible influence of slight eye movement under topical anesthesia is trivial; however, the actual mechanism should be studied further.

Harder et $\mathrm{al}^{18}$ conducted a prospective study comparing an intravitreal bevacizumab injection group for ROP threshold disease in posterior zone 2 or zone 1 or for prethreshold ROP in zone 1 with a control group that had previously undergone argon laser therapy. They found that the mean refractive errors of both eyes were significantly less myopic in the bevacizumab group than in the laser group at a mean corrected age of 10.5 months. Furthermore, in a 1-year follow-up study, they concluded that a single intravitreal bevacizumab injection led to less myopization and less astigmatism than the conventional retinal laser therapy. ${ }^{19}$ In our study, we also found that a single intravitreal ranibizumab or bevacizumab injection led to less myopization.

However, Tseng et $\mathrm{al}^{20}$ reported substantial refractive errors possibly resulting from an intravitreal bevacizumab injection in one of triplets. The triplet presented with threshold ROP and was treated with intravitreal bevacizumab in both eyes. Substantial refractive errors up to $-9.75 \mathrm{D}$ in the right eye and $-11.5 \mathrm{D}$ in the left eye were noted at the 14-month follow-up visit. The refractive change in this case may be a possible complication related to intravitreal bevacizumab treatment. It is uncertain whether bevacizumab influenced the development of myopia in a different manner than in other patients with ROP; however, this case serves as a warning to clinicians that careful follow-up of refraction is important in patients treated with intravitreal bevacizumab.

In this study, there were no significant differences in the axial length and refraction between those who received intravitreal bevacizumab and those who received intravitreal ranibizumab after 1 year of follow-up. Therefore, bevacizumab and ranibizumab may influence ocular development in a similar manner. Incomplete retinal vascularization toward the retinal periphery was noted in ten of $25(40 \%)$ eyes of the ranibizumab group and eight of $15(53.3 \%)$ eyes of the bevacizumab group after 1 year of treatment. It seemed that the ranibizumab treatment could achieve more complete retinal vascularization than the bevacizumab treatment; however, there was no statistical significance.

Christoforidis et $\mathrm{al}^{21}$ found that intravitreal retention lasted longer for bevacizumab than ranibizumab in a rabbit model. The rabbit vitreous volume is closer in size to that of an ROP eye (1.6-2.5 mL) ${ }^{22}$ and may be used to more accurately simulate the intravitreal pharmacokinetic properties of these two agents in premature eyes. We could assume that ranibizumab also has shorter intravitreal VEGF suppression in the eyes of premature babies. This might be a possible explanation why ranibizumab treatment could achieve more complete normal retinal vascularization than the bevacizumab treatment. In such ROP cases receiving bevacizumab or ranibizumab treatment, long-term fundus examinations are mandatory for early detection of deterioration and timely intervention. ${ }^{2}$

The limitations of this study include its retrospective nature and the small sample size. Owing to the limited number of cases in our series, we were not looking forward to omitting any treated eyes. After full discussion with our statistical board, we figured out a powerful statistical method called GLMM to analyze this kind of clustered data (both eyes from the same patient in either group in our cases). 
This kind of method can remove the random effect due to the dependency of data caused from the patient factor. PROC GLIMMIX in SAS, which works under the principle of GLMM, was used to replace the $t$-test or Fisher's test to analyze the data. In addition, fundus photographs were not available in all cases. The main disadvantage of ranibizumab treatment is a higher expense than bevacizumab.

\section{Conclusion}

There were no significant differences in the axial length and refraction between the children who received intravitreal bevacizumab and those who received intravitreal ranibizumab after 1 year of follow-up. Nevertheless, incomplete normal retinal vascularization toward the retinal periphery was still noted in approximately half of the cases. Therefore, long-term follow-up of ROP patients who have received bevacizumab or ranibizumab treatment is needed for early detection of deterioration and timely intervention.

\section{Disclosure}

The authors report no conflicts of interest in this work. The authors do not have any commercial or proprietary interest in the products or company.

\section{References}

1. International Committee for the Classification of Retinopathy of Prematurity. The international classification of retinopathy of prematurity revisited. Arch Ophthalmol. 2005;123(7):991-999.

2. Mintz-Hittner HA, Kennedy KA, Chuang AZ; BEAT-ROP Cooperative Group. Efficacy of intravitreal bevacizumab for stage 3+ retinopathy of prematurity. N Engl J Med. 2011;364(7):603-615.

3. Wu WC, Yeh PT, Chen SN, Yang CM, Lai CC, Kuo HK. Effects and complications of bevacizumab use in patients with retinopathy of prematurity: a multicenter study in Taiwan. Ophthalmology. 2011;118(1): 176-183.

4. Wu WC, Kuo HK, Yeh PT, Yang CM, Lai CC, Chen SN. An updated study of the use of bevacizumab in the treatment of patients with prethreshold retinopathy of prematurity in Taiwan. Am J Ophthalmol. 2013; 155(1):150-158.

5. Martínez-Castellanos MA, Schwartz S, Hernández-Rojas ML, et al. Long-term effect of antiangiogenic therapy for retinopathy of prematurity up to 5 years of follow-up. Retina. 2013;33(2):329-338.

6. Orozco-Gómez LP, Hernández-Salazar L, Moguel-Ancheita S, RamírezMoreno MA, Morales-Cruz MV. Laser-ranibizumab treatment for retinopathy of prematurity in umbral-preumbral disease. Three years of experience. Cir Cir. 2011;79(3):207-214, 225-232.

Clinical Ophthalmology

\section{Publish your work in this journal}

Clinical Ophthalmology is an international, peer-reviewed journal covering all subspecialties within ophthalmology. Key topics include: Optometry; Visual science; Pharmacology and drug therapy in eye diseases; Basic Sciences; Primary and Secondary eye care; Patient Safety and Quality of Care Improvements. This journal is indexed on
7. Lin CJ, Chen SN, Tseng CC, Chang YC, Hwang JF. Effects of ranibizumab on very low birth weight infants with stage 3 retinopathy of prematurity: a preliminary report. Taiwan J Ophthalmol. 2012;2(4):136-139.

8. Cryotherapy for Retinopathy of Prematurity Cooperative Group. Multicenter trial of cryotherapy for retinopathy of prematurity: preliminary results. Arch Ophthalmol. 1988;106(4):471-479.

9. Early Treatment for Retinopathy of Prematurity Cooperative Group. Revised indications for the treatment of retinopathy of prematurity: results of the early treatment for retinopathy of prematurity randomized trial. Arch Ophthalmol. 2003;121(12):1684-1694.

10. Quinn GE, Dobson V, Siatkowski R, et al. Does cryotherapy affect refractive error? Results from treated versus control eyes in the cryotherapy for retinopathy of prematurity trial. Ophthalmology. 2001; 108(2):343-347.

11. Connolly BP, Ng EY, McNamara JA, Regillo CD, Vander JF, Tasman W. A comparison of laser photocoagulation with cryotherapy for threshold retinopathy of prematurity at 10 years, part 2: refractive outcome. Ophthalmology. 2002;109(5):936-941.

12. Davitt BV, Dobson V, Good WV, et al. Prevalence of myopia at 9 months in infants with high-risk prethreshold retinopathy of prematurity. Ophthalmology. 2005;112(9):1564-1568.

13. Katoch D, Sanghi G, Dogra MR, Beke N, Gupta A. Structural sequelae and refractive outcome 1 year after laser treatment for type 1 prethreshold retinopathy of prematurity in Asian Indian eyes. Indian J Ophthalmol. 2011;59(6):423-426.

14. Davitt BV, Quinn GE, Wallace DK, et al. Astigmatism progression in the early treatment for retinopathy of prematurity study to 6 years of age. Ophthalmology. 2011;118(12):2326-2329.

15. Sato T, Kusaka S, Shimojo H, Fujikado T. Vitreous levels of erythropoietin and vascular endothelial growth factor in eyes with retinopathy of prematurity. Ophthalmology. 2009;116(9):1599-1603.

16. Jang SY, Choi KS, Lee SJ. Delayed-onset retinal detachment after an intravitreal injection of ranibizumab for zone 1 plus retinopathy of prematurity. J AAPOS. 2010;14(5):457-459.

17. Lin CJ, Chen SN, Hwang JF. Intravitreal ranibizumab as salvage therapy in an extremely low-birth-weight infant with rush type retinopathy of prematurity. Oman J Ophthalmol. 2012;5(3):184-186.

18. Harder BC, von Baltz S, Schlichtenbrede FC, Jonas JB. Early refractive outcome after intravitreous bevacizumab for retinopathy of prematurity. Arch Ophthalmol. 2012;130(6):800-801.

19. Harder BC, Schlichtenbrede FC, von Baltz S, Jendritza W, Jendritza B, Jonas JB. Intravitreal bevacizumab for retinopathy of prematurity: refractive error results. Am J Ophthalmol. 2013;155(6):1119-1124.

20. Tseng CC, Chen SN, Hwang JF, Lin CJ. Different refractive errors in triplets with retinopathy of prematurity treated with bevacizumab. $J$ Pediatr Ophthalmol Strabismus. 2012;49:e41-e43.

21. Christoforidis JB, Williams MM, Wang J, et al. Anatomic and pharmacokinetic properties of intravitreal bevacizumab and ranibizumab after vitrectomy and lensectomy. Retina. 2013;33(5):946-952.

22. Meyer CH, Krohne TU, Holz FG. Intraocular pharmacokinetics after a single intravitreal injection of $1.5 \mathrm{mg}$ versus $3.0 \mathrm{mg}$ of bevacizumab in humans. Retina. 2011;31(9):1877-1884.

PubMed Central and CAS, and is the official journal of The Society of Clinical Ophthalmology (SCO). The manuscript management system is completely online and includes a very quick and fair peer-review system, which is all easy to use. Visit http://www.dovepress.com/ testimonials.php to read real quotes from published authors. 